\title{
A new possibility for production of sub-picosecond x-ray pulses using a time dependent radio frequency orbit deflection
}

\author{
A. Zholents ${ }^{1}$ \\ ${ }^{1}$ Argonne National Laboratory, Argonne, Illinois 60439, USA
}

\begin{abstract}
It is shown that two radio frequency deflecting cavities with slightly different frequencies can be used to produce time-dependent orbit deflection to a few special electron bunches circulating in a synchrotron without affecting the majority of the electron bunches. These special bunches produce an x-ray pulse in which transverse position or angle, or both, are correlated with time. The x-ray pulse is then shortened, either with an asymmetrically cut crystal that acts as a pulse compressor, or with an angular aperture such as a narrow slit positioned downstream. The implementation of this technique creates a highly flexible environment for synchrotrons in which users of most beamlines will be able to easily select between the x-rays originated by the standard electron bunches and the short $\mathrm{x}$-ray pulses originated by the special electron bunches carrying a time-dependent transverse correlation.
\end{abstract}

PACS numbers:

\section{Introduction}

X-ray pulses with a few tens of femtoseconds pulse duration are routinely available at free-electron laser facilities FLASH [1], LCLS [2], SACLA[3], Fermi@Elletra[4] for time resolved studies of matter. However a demand is growing for investigations of reversible dynamics in molecular systems and materials in a non-destructive way at synchrotrons[5-10]. To compensate for a significantly reduced peak flux of photons, the synchrotrons employ a high repetition rate of x-ray pulses up to hundreds of $\mathrm{MHz}$. Since the speed of sound in materials is $\sim 1$ $\mathrm{nm} / \mathrm{ps}$, in many cases the natural timescale to probe charge transfer dynamics, orbital, spin and lattice degrees of freedom in materials on the nanometer length scale is a picosecond. This is why several synchrotrons use a low alpha lattice [11-13] to shorten the electron bunches and therefore produce $\mathrm{x}$-ray pulses from tens of picoseconds to a few picoseconds. A significant shortening of the electron bunches by means of a high frequency and high voltage radio frequency (rf) accelerating system has also been considered [14, 15].

Obtaining sub-picosecond x-ray pulses without compression of the electron bunches is also possible. For example, laser slicing[16-18] and electron beam slicing[19] allow selection of only a small fraction of the x-ray pulse emitted by a long electron bunch. The other way to select short $\mathrm{x}$-ray pulses is with a pair of rf deflecting cavities [20, 21]. Specifically, the first rf deflecting cavity imposes a time-dependent angular kick to the electron bunch. Then the beam is sent through undulators and bending magnets to produce an x-ray pulse in which transverse position or angle is also correlated with time. The x-ray pulse is then further shortened, either with an asymmetrically cut crystal that acts as a pulse compressor, or with an angular aperture such as a narrow slit positioned downstream. The second rf deflecting cavity then cancels the initial spatial chirp on the electron bunch, minimizing perturbations to the beam dynamics in the rest of the ring. Although every electron bunch is affected, chirp cancellation preserves normal operation for the majority of beamlines and leaves only a handful of beamlines located between the two rf deflecting cavities to operate with the short x-ray pulses. These beamlines typically trade $\mathrm{x}$-ray beam brightness for a shorter pulse duration.

Here we propose a new modification of this technique in which many beamlines can select between using either high brightness long x-ray pulses from standard electron bunches or short x-ray pulses with a reduced brightness from a handful number of special electron bunches with an imposed time-dependent transverse correlation.

\section{Method}

The desired result can be achieved by employing two rf deflecting cavities with slightly different frequencies $f_{1}$ and $f_{2}$ in a similar approach as taken in Ref [15] for production of a fill pattern with an alternating bunch length:

$$
\begin{aligned}
& f_{1}=h f_{R F} \\
& f_{2}=\frac{h m \pm 1}{m} f_{R F} .
\end{aligned}
$$

Here $f_{R F}$ is the frequency of the main $\mathrm{rf}$ system, $h$ is the harmonic number and $m=f_{R F} /\left(N f_{0}\right)$ is the number of main rf separatrices between two neighboring standard electron bunches, $f_{0}$ is the revolution frequency and $N$ is the number of standard electron bunches uniformly distributed in the storage ring. The separation between the standard electron bunch and the special electron bunch 
must be $m / 2$ separatrices for optimal kick of the special bunch. Two rf cavities can be phased to cancel a time dependent kick produced by each system in all separatrices occupied by the standard electron bunches, e.g.:

$$
\delta y^{\prime}(\hat{z})=\frac{e U_{1}}{E_{b}} \sin \left(2 \pi f_{1} \sigma_{\tau} \hat{z}\right)-\frac{e U_{2}}{E_{b}} \sin \left(2 \pi f_{2} \sigma_{\tau} \hat{z}\right),
$$

where $e$ is the electron charge, $U_{1}$ and $U_{2}$ are cavity 'transverse' voltages, $E_{b}$ is the electron beam energy, $c \sigma_{\tau}$ is the r.m.s. electron bunch length, $c$ is the speed of light and $\hat{z}$ is the distance to the bunch center normalized on $c \sigma_{\tau}$. A condition of $U_{2}=U_{1}$ cancels the kick for the central slice of the electron bunch, but better cancellation weighted over all slices is when $U_{2}$ is slightly different, e.g., $U_{2}=U_{1}(1+u)$, where $u$ can be found from minimization of the r.m.s. value of the residual kick $\sigma_{r e s}$ :

$$
u \approx \frac{e^{-\frac{1}{2}\left(a_{1}+a_{2}\right)^{2}}\left(e^{2 a_{1} a_{2}}-1\right)}{1-e^{-2 a_{2}^{2}}}-1,
$$

where $a_{1}=2 \pi f_{1} \sigma_{\tau}, a_{2}=2 \pi f_{2} \sigma_{\tau}$ and

$$
\sigma_{r e s} \approx \frac{e U_{1}\left|a_{1}-a_{2}\right|}{\sqrt{2} E_{b}}\left(\frac{4 a_{1}^{2}+e^{-2 a_{1}^{2}}-e^{2 a_{1}^{2}}}{1-e^{2 a_{1}^{2}}}\right)^{1 / 2} .
$$

This is strictly valid only for a Gaussian distribution of electron density in $z$. For a minimum residual distortion, the value of $\sigma_{\text {res }}$ should be much less than the beam angular spread in the vertical direction, in which case the residual distortion will mostly be caused by errors in the rf fields.

At the same time, two cavities will cooperate producing a strong time-dependent angular kick to the electron bunches located in the separatrices shifted by $m / 2$ separatrices from the standard bunches:

$$
\begin{aligned}
\delta y^{\prime}(\hat{z}) & =\frac{e U_{1}}{E_{b}}\left(\sin \left(a_{1} \hat{z}\right)+(1+u) \sin \left(a_{2} \hat{z}\right)\right) \\
& \approx 2 \frac{e U_{1}}{E_{b}} \sin \left(a_{1} \hat{z}\right)
\end{aligned}
$$

where the approximation is valid for $u<<1$ and $f_{2} \approx f_{1}$ corresponding to the above assumptions. The repetitive kicks applied to the electron bunch on each orbit turn produce a closed orbit distortion around the ring with the angle and displacement in the center of the deflecting cavity equal to:

$$
\begin{aligned}
& y_{0}^{\prime}(\hat{z})=\frac{1}{2} \delta y^{\prime}(\hat{z}) \\
& y_{0}(\hat{z})=\frac{1}{2} \beta \delta y^{\prime}(\hat{z}) \cot (\pi \nu) .
\end{aligned}
$$

Here $\nu$ is the vertical betatron tune. For simplicity we assume zero length deflecting cavities located in the center of the straight section where the vertical beta-function is equal to $\beta$ and has a zero derivative. We also assume that the storage ring lattice consists of identical sectors with the phase advance $\phi$ of the vertical betatron oscillations between two neighboring sectors and find the electron bunch chirp in all other sectors of the storage ring using Eqs. (5,6):

$$
\begin{aligned}
y^{\prime}(\hat{z}) & =-\frac{e U_{1}}{E_{b}} \sin \left(a_{1} \hat{z}\right) \frac{\sin (k \phi-\pi \nu)}{\sin (\pi \nu)} \\
y(\hat{z}) & =\beta \frac{e U_{1}}{E_{b}} \sin \left(a_{1} \hat{z}\right) \frac{\cos (k \phi-\pi \nu)}{\sin (\pi \nu)},
\end{aligned}
$$

where $k$ is the sector number counting from the sector with the deflecting cavities. The most convenient chirp for production of the short x-ray pulses using undulators is when $y(\hat{z}) \approx 0$ and $y^{\prime}(\hat{z})$ is at the maximum, e.g., when $k \phi-\pi \nu \approx \pi\left(j+\frac{1}{2}\right)$, where $j$ is an integer.

The accelerating fields of the deflecting cavities [20] cause a small distortion in the longitudinal electron motion that does not lead to any detrimental effects. However because of this field, it is important to keep the electron beam at the cavity center in the vertical plane where the accelerating field changes sign, in which case there will be no energy exchange between the electron beam and the cavity field, at least for the electron bunches with a symmetrical electron density distribution in $z$. Even if the distribution is slightly asymmetrical, a small orbit offset in $y$ could still keep the energy exchange balance close to zero.

Using the angular chirp defined in Eq. (7), the short $\mathrm{x}$-ray pulse is obtained in the x-ray beamline, either with an asymmetrically cut crystal that acts as a pulse compressor, or with an angular aperture such as a narrow slit positioned downstream from the source. Following [20] we define the r.m.s. duration of the x-ray pulse as:

$$
\sigma_{x-\text { ray }} \approx \sigma_{\tau} \frac{\sqrt{\sigma_{y^{\prime}}^{2}+\sigma_{\theta}^{2}}}{y^{\prime}(1)}
$$

where $\sigma_{y^{\prime}}=\sqrt{\epsilon_{y} / \beta}$ is the r.m.s. electron beam divergence, $\epsilon_{y}$ is the vertical emittance, and $\sigma_{\theta}=\sqrt{\frac{\lambda_{x}}{\pi L_{u}}}$ is the r.m.s. divergence of the undulator radiation at the wavelength $\lambda_{x}[22]$, and $L_{u}$ is the undulator length.

Having a small $\sigma_{\theta}$ simplifies obtaining the smallest possible $\sigma_{x-\text { ray }}$. Therefore, we assume that one long undulator occupies the entire straight section and that the storage ring lattice uses an optimal $\beta \approx L_{u} / 2$, in which case the average vertical beam size in the undulator is at a minimum. Using Eq. (7), we find the maximum displacement of the electrons at the end of the undulator:

$$
\Delta y(\hat{z})=\frac{L_{u} e U_{1}}{\sqrt{2} E_{b} \sin (\pi \nu)} \cos \left(k \phi-\pi \nu+\frac{\pi}{4}\right) \sin \left(a_{1} \hat{z}\right)
$$

This displacement should not exceed the beam-stay-clear aperture $a_{y}$. Thus, one finds the maximum attainable 
$y^{\prime}(1)$ :

$$
y^{\prime}(\hat{z}=1)_{\max }=\sqrt{2} \frac{a_{y}}{L_{u}} \sin \left(a_{1}\right)
$$

Using (10) in Eq. (8), one obtains for the x-ray pulse duration constrained by the beam-stay-clear aperture:

$$
\sigma_{x-\text { ray }} \approx \frac{\sqrt{\epsilon_{y} \frac{L_{u}}{2}+\frac{\lambda_{x} L_{u}}{4 \pi}}}{\sqrt{2} a_{y} \pi f_{1}} .
$$

Achieving it would require:

$$
U_{1}=\frac{E_{b}}{e} \frac{a_{y}}{L_{u}} \sqrt{2}|\sin (\pi \nu)|
$$

However, if this 'transverse' voltage cannot be provided by the cavity, then the x-ray pulse duration will be constrained by the attainable 'transverse' voltage:

$$
\sigma_{x-\text { ray }} \approx \frac{E_{b}}{e U_{1}} \frac{\sqrt{\frac{2 \epsilon_{y}}{L_{u}}+\frac{\lambda_{x}}{\pi L_{u}}}}{2 \pi f_{1}}|\sin (\pi \nu)| .
$$

Finally, having $y(\hat{z}) \approx 0$ in the undulator is convenient, but not absolutely necessary. In principle, any electron bunch chirp defined by Eq. (7) can be utilized for a selection of short x-ray pulses providing that the $\mathrm{x}$-ray optics is optimized in each case. For example, even slightly shorter x-ray pulses can be obtained when the chirp is such that $y(\hat{z})$ is at the maximum and $y^{\prime}(\hat{z}) \approx 0$ :

$$
\sigma_{x-r a y} \approx \frac{E_{b}}{e U_{1}} \frac{\sqrt{\frac{2 \epsilon_{y}}{L_{u}}+\frac{\lambda_{x}}{4 \pi L_{u}}}}{2 \pi f_{1}}|\sin (\pi \nu)|,
$$

in which case the $\mathrm{x}$-ray mirrors must be used to create an intermediate focusing where selection of a short x-ray pulse can be made. A non-specular reflection from the $\mathrm{x}$-ray mirrors will have a tendency to degrade the signal to noise ratio and should be minimized using high quality mirrors.

\section{Degrading effects}

There are two effects that degrade the vertical emittance in the special electron bunches and, thus, the x-ray pulse duration according to Eqs. $(11,13,14)$.

A typical storage ring lattice contains a large number of sextupole magnets that are used to control chromaticity. When an electron is displaced in a sextupole in the vertical and horizontal directions it receives a kick in the vertical plane that is proportional to the electron's horizontal coordinate $x$, e.g., $\delta y^{\prime} \simeq b_{s} y(\hat{z}) x$, where $b_{s}$ is the normalized strength of the sextupole. This kick couples a vertical electron motion to a horizontal electron motion. A combination of all these kicks from all storage ring sextupoles can increase a width of the linear coupling resonance and produce a noticeable increase of the vertical emittance, in particular when the betatron tunes are in a close proximity to the resonance line. However, it has been shown in [23] that one can optimize sextupole's strengths and achieve a mutual compensation of all vertical kicks while keeping the same chromaticity as before. In this case, no emittance increase due to coupling is expected.

The second effect takes its roots in quantum fluctuations of synchrotron radiation. It can be understood by comparing synchrotron oscillations of the electron with and without quantum fluctuations. Without the fluctuations, the arriving time of the electron in the deflecting cavities on each orbit turn follows a regular pattern and so does the vertical kick that this electron receives in the deflecting cavities. With the fluctuations, the arriving time fluctuates by a small amount and it causes some randomization in the magnitude of the vertical kicks. A typical error in the kick is

$$
\delta \tilde{y^{\prime}}=2 \frac{e U_{1}}{E_{b}} \cos \left(a_{1} \hat{z}\right) a_{1} \Delta \hat{z}
$$

where $\Delta \hat{z}$ is the error in the arriving time normalized on the electron bunch length. It is caused by a fluctuation of the actual places where photons are radiated and also by a fluctuation of the actual amount of the electron energy loss for the radiation on the orbit turn. The orbit kick errors (15) are uncorrelated on each orbit turn and lead to a diffusive growth of the vertical beam emittance. The synchrotron radiation damping counter-acts this growth such that in equilibrium the following addition to the vertical emittance is produced: $\tilde{\epsilon_{y}}=\frac{L_{u}}{2}\left\langle\left\langle\delta{\tilde{y^{\prime}}}^{2}\right\rangle\right\rangle$. Here the first average is taken over the error in the arrival time using $\left\langle\Delta \hat{z}^{2}\right\rangle=T / \tau_{d}$, where $T$ is the revolution period and $\tau_{d}$ is the synchrotron radiation damping time, and the second average is taken over the distribution of electrons in $\hat{z}$. The result is:

$$
\tilde{\epsilon_{y}} \approx \frac{L_{u}}{2}\left(2 \frac{e U_{1}}{E_{b}} a_{1}\right)^{2} \frac{T}{\tau_{d}} e^{-a_{1}^{2}} \cosh \left(a_{1}^{2}\right) .
$$

One can see that $\tilde{\epsilon_{y}}$ only weakly depends on $f_{1}$ while $\sigma_{x-\text { ray }}$ has a rather strong dependence on $f_{1}$. Therefore, the preference is for operating deflecting cavities at a high frequency.

The shortest $\mathrm{x}$-ray pulse that can be achieved with this method in the limit of $\frac{\lambda_{x}}{4 \pi}, \epsilon_{y}<<\tilde{\epsilon_{y}}$ can be written using Eqs. (13,16) as:

$$
\tilde{\sigma}_{x-\text { ray }}=2 \sigma_{\tau}\left(\frac{T}{\tau_{d}} e^{-a_{1}^{2}} \cosh \left(a_{1}^{2}\right)\right)^{1 / 2}|\sin (\pi \nu)| .
$$




\section{Numerical examples}

The analysis presented above is illustrated in this section with numerical examples using typical parameters that can be obtained at a hard x-ray source such as the APS [24] and a soft x-ray source such as the BESSY-II [25]. The APS operates with $f_{R F}=352 \mathrm{MHz}$ and with 24 bunches uniformly distributed around the ring with $m=54$. Thus, using $h=8$ in Eq. (1), one obtains $f_{1}=2816$ $\mathrm{MHz}$ and $f_{2}=2822.52 \mathrm{MHz}$, in which case the special separatrices where bunch tilting can take place are located in the middle between two standard bunches. In the case of the BESSY-II with the standard bunches occupying every second separatrix and $f_{R F}=500 \mathrm{MHz}$, selection of $h=6$ leads to $f_{1}=3000 \mathrm{MHz}$ and $f_{2}=2750 \mathrm{MHz}$. Obviously, depending on the experimental needs, only a few of the special separatrices can actually be populated with electrons. It is also worth noting that the choice of high frequencies for $f_{1}$ and $f_{2}$ serves a double purpose, i.e., it helps to obtain shorter x-ray pulses as per Eqs. $(11,13)$ and to contain the maximum excursion of $\Delta y$ within the available beam-stay-clear aperture (e.g., see Figure 1). All parameters required for calculation of $\sigma_{x-\text { ray }}$ and other quantities are given in Table I.

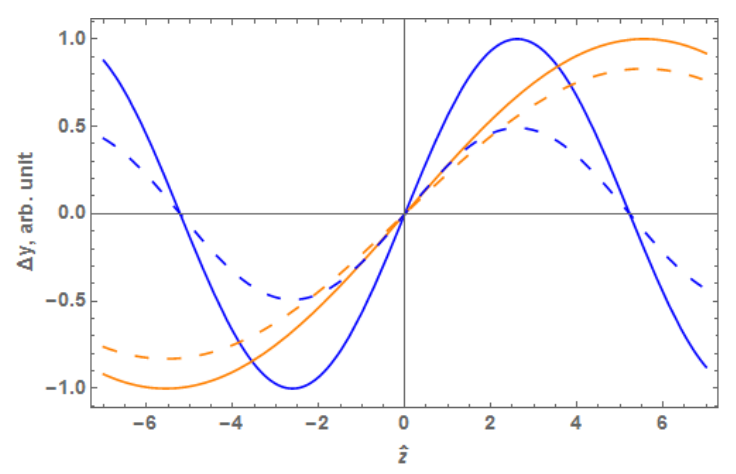

FIG. 1: The displacement $\Delta y$ of the electrons at the end of the undulator as a function of $\hat{z}$ in the case of the APS and BESSY (blue and orange curves). The upper and lower boundaries represent the beam-stay-clear apertures. The range for $\hat{z}$ from -7 to 7 accounts for the tails in the longitudinal distribution important for maintaining a good beam lifetime, whereas $99.7 \%$ of electrons are contained in the range from -3 to 3 . In practice, the maximum $\Delta y$ is limited by the attainable 'transverse' voltage. It is shown here for comparison with dashed curves.

A practical design of a compact superconducting rf deflecting cavity operating at $f_{1}=2815 \mathrm{MHz}$ and capable of a $2 \mathrm{MV}$ deflecting voltage is described in [26, 27].

Figures (2) and (3) show projected $\sigma_{x-\text { ray }}$ for the case of the hard and soft x-ray source as a function of the photon energy. As expected, in the hard x-ray case, the diffraction of the x-rays is the limiting factor at the lower energy end while the electron beam emittance is the limiting factor at the higher energy end. Two curves in
TABLE I: Example parameters for APS and BESSY-II.

\begin{tabular}{cccc}
\hline Parameter & APS & BESSY-II & Units \\
\hline$E_{b}$ & 7 & 1.7 & $\mathrm{GeV}$ \\
$\epsilon_{y}$ & 20 & 20 & $\mathrm{pm}$ \\
$L_{u}$ & 5 & 4 & $\mathrm{~m}$ \\
$a_{y}$ & 3.5 & 5.5 & $\mathrm{~mm}$ \\
$\nu$ & 19.2 & 6.74 & \\
$\sigma_{\tau}$ & 34 & 15 & $\mathrm{ps}$ \\
$f_{1}$ & 2816 & 3000 & $\mathrm{MHz}$ \\
$U_{1}$ & 2 & 2 & $\mathrm{MV}$ \\
$\delta \tilde{\epsilon}_{y}$ & 84 & 40 & $\mathrm{pm}$ \\
$\mathrm{u}$ & $-1.610^{-3}$ & $8.410^{-2}$ & \\
$\sigma_{\text {res }}$ & $9.810^{-8}$ & $1.710^{-6}$ & \\
$\lambda_{x}$ & 0.1 & 4.4 & $\mathrm{~nm}$ \\
$\sigma_{x-\text { ray }}$ & $0.8^{*}$ & $0.6^{*}$ & $\mathrm{ps}$ \\
$\tilde{\sigma}_{x-\text { ray }}$ & 0.67 & 0.12 & $\mathrm{ps}$ \\
\hline * constrained by 'transverse' voltage
\end{tabular}

Fig. (2) correspond to nominal emittances of $40 \mathrm{pm}$ and $20 \mathrm{pm}$. Further emittance reduction will continue to drive $\sigma_{x-\text { ray }}$ a little down until it will reach the limiting case. In the soft x-ray case, the diffraction of the x-rays always limits the attainable pulse duration and lowering of the emittance does not help much as seen in Fig. (3).

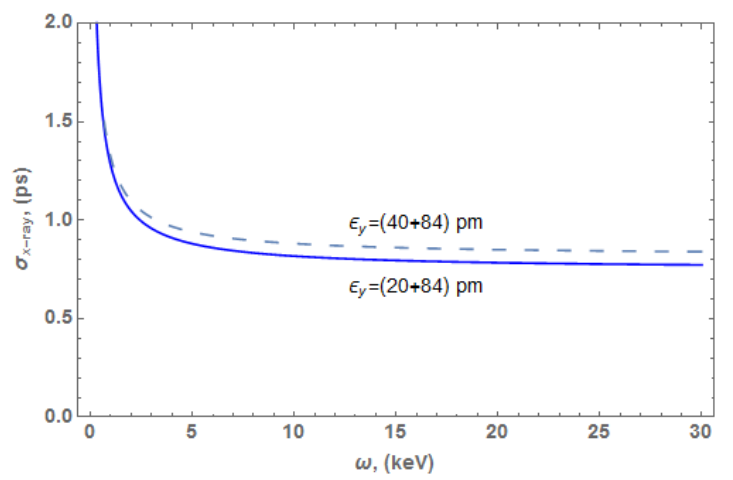

FIG. 2: A projected duration of the hard x-ray pulses at the APS as a function of the photon energy. The emittance is defined as a sum of $\epsilon_{y}$ and $\tilde{\epsilon_{y}}$.

Figure (4) shows the result of a particle tracking including effects of the quantum fluctuation and synchrotron radiation damping using elegant [28] for the APS lattice and parameters from Table I. The scattered plot of electrons is given for the straight section where the bunch angular chirp is at the maximum. 


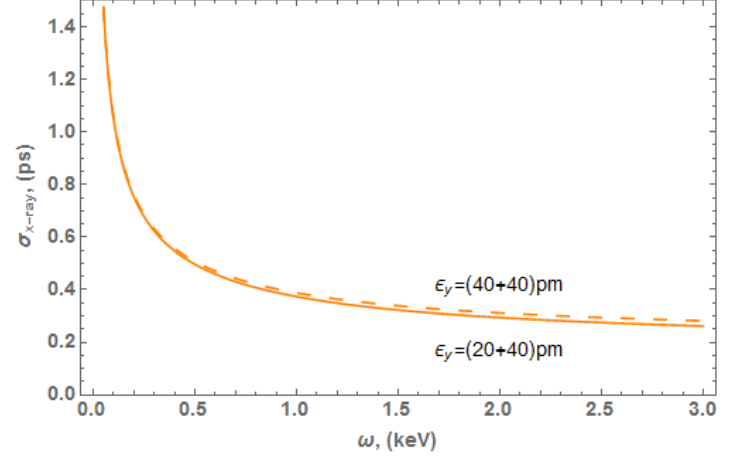

FIG. 3: A projected duration of the soft x-ray pulses at the BESSY-II as a function of the photon energy. The emittance is defined as a sum of $\epsilon_{y}$ and $\tilde{\epsilon_{y}}$.

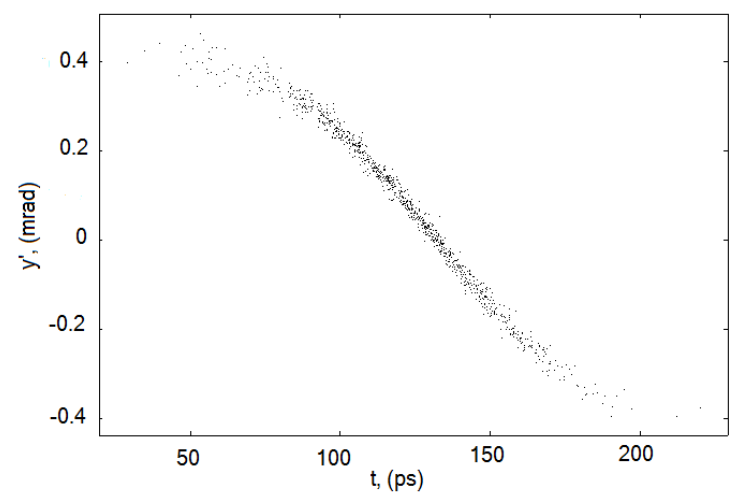

FIG. 4: The illustration of the angular chirp of the electron bunch obtained by a particle tracking using elegant [28].

\section{Selection of short x-ray pulses}

A separation of the stand alone short x-ray pulses from the main stream radiation produced by the standard electron bunches is relatively easy for the technique discussed in this paper. For simplicity consider the straight section undulator where $y(\hat{z})$ of the special electron bunch is close to zero for all $\hat{z}$ and the angular chirp $y^{\prime}(\hat{z})$ is large. When the standard electron bunch passes the undulator, all electrons radiate predominantly forward as shown schematically in Fig. (5a). However, when the special electron bunch passes the undulator, different slices of the electron bunch radiate in different directions (see Fig. (5b) ). Thus, any direction away from the main stream radiation can be used to select a stand alone $\mathrm{x}$-ray pulse. Alternatively, the main stream radiation can be sent with an angle as shown in Fig. (5c) using a local beam orbit zigzag, in which case the forward direction will receive short x-ray pulses (see Fig. (5d)). In most cases providing an orbit angle that is slightly larger than the opening angle of the undulator radia- tion convoluted with the angular spread of electrons, i.e., $\theta \approx \sqrt{2 \pi} \sqrt{\sigma_{\theta}^{2}+\sigma_{y^{\prime}}^{2}}$, should ensure a good signalto-background ratio for short $\mathrm{x}$-ray pulses.

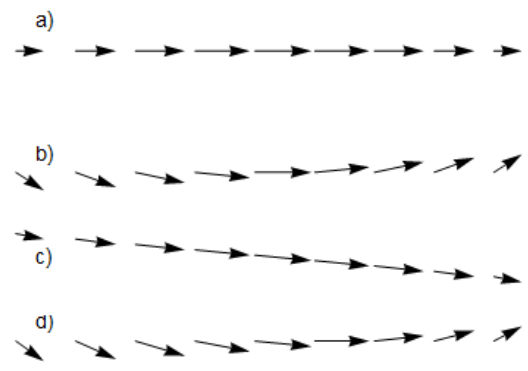

FIG. 5: The illustration of the idea for separation of the short $\mathrm{x}$-ray pulses: a) the radiation pattern of the standard electron bunch, b) the radiation pattern of the special electron bunch with different slices pointing into different directions, c) the radiation pattern of the standard electron bunch with an orbit angle, d) the radiation pattern of the special electron bunch with an orbit angle.

\section{ERRORS}

The amplitude and phase errors in the rf fields of the deflecting cavities will only slightly affect the timedependent orbit deflection of the special electron bunches, but they will distort a perfect balance of the rf fields experienced by the standard electron bunches and expose them to similar time-dependent orbit deflections as arranged for the special electron bunches, though with a much smaller magnitude. An accurate assessment of the impact of these deflections on the properties of the x-ray radiation produced by standard electron bunches requires rigorous computer modeling that is out of the scope of this paper. Instead, we assess the sensitivities to errors in two characteristic cases. In the first case we consider errors at a high frequency end of the spectrum above the revolution frequency. In this case, the orbit kicks driven by errors are uncorrelated on each orbit turn and lead to a diffusive growth of the vertical beam emittance. The synchrotron radiation damping counter-acts this growth such that in equilibrium the following addition to the vertical emittance is produced:

$$
\begin{aligned}
\Delta \epsilon_{y}^{(1)} \approx & 2\left(\frac{e U_{1}}{E_{b}}\right)^{2} \frac{\tau_{d}}{T} \frac{L_{u}}{2} e^{-a_{1}^{2}} \\
& \left(\sinh \left(a_{1}^{2}\right) \sigma_{U}^{2}+\cosh \left(a_{1}^{2}\right) \sigma_{\phi}^{2}\right) .
\end{aligned}
$$

Here $\sigma_{U}$ is the relative error in the 'transverse' voltage of the deflecting cavity, $\sigma_{\phi}$ is the phase error of the rf phase of the deflecting cavity, and $\beta=L_{u} / 2$ is assumed. The errors in both cavities are considered to be uncorrelated. Deriving Eq.(18), we applied averaging over the electron 
density distribution in $z$. A reduction of the primary vertical emittance using a smaller coupling to the horizontal emittance may help to offset the above defined emittance increase.

In the second case we consider a low frequency end of the error spectrum with a relevant time scale comparable to or longer than the damping time. In this case, the repetitive orbit kicks experienced by electron bunches during a large number of orbit turns create slowly changing, but more or less sustainable chirped bunch patterns in all machine sectors similar to the ones described by Eq.(6), though with a much smaller magnitude. These coordinate and angular chirps increase the effective beam size and divergence that can be conveniently characterized by the effective vertical emittance:

$$
\begin{aligned}
\Delta \epsilon_{y}^{(2)} \approx & 2\left(\frac{e U_{1}}{E_{b}}\right)^{2} \frac{L_{u}}{2 \sin \pi \nu^{2}} e^{-a_{1}^{2}} \\
& \left(\sinh \left(a_{1}^{2}\right) \sigma_{U}^{2}+\cosh \left(a_{1}^{2}\right) \sigma_{\phi}^{2}\right) .
\end{aligned}
$$

Using typical parameters that can be obtained at a hard x-ray source such as the APS and a soft x-ray source such as the BESSY-II we demonstrate sensitivities to errors in the high frequency range in Table (II) and low frequency range in Table (III). In both cases we select $\sigma_{U}$ and $\sigma_{\phi}$ such as they contribute equally to emittance increase.

TABLE II: High frequency errors.

\begin{tabular}{cccc}
\hline Parameter & APS & BESSY-II & Units \\
\hline$\tau_{d}$ & 9.6 & 16 & $\mathrm{~ms}$ \\
$T$ & 3.7 & 0.8 & $\mu \mathrm{s}$ \\
$\sigma_{U}$ & $1.510^{-4}$ & $410^{-5}$ & \\
$\sigma_{\phi}$ & $510^{-3}$ & $610^{-4}$ & Degree \\
$\Delta \epsilon_{y}^{(1)}$ & 12 & 24 & $\mathrm{pm}$ \\
\hline
\end{tabular}

TABLE III: Low frequency errors.

\begin{tabular}{cccc}
\hline Parameter & APS & BESSY-II & Units \\
\hline$\sigma_{U}$ & $1.510^{-3}$ & $410^{-3}$ & \\
$\sigma_{\phi}$ & $510^{-2}$ & $6.510^{-2}$ & Degree \\
$\Delta \epsilon_{y}^{(2)}$ & 13 & 24 & pm \\
\hline
\end{tabular}

\section{CONCLUSION}

The technique for production of picosecond $\mathrm{x}$-ray pulses on multiple beamlines of synchrotrons using two rf deflecting cavities with slightly different frequencies has been presented and analyzed. The implementation of this technique creates a highly flexible environment for synchrotrons in which users of most beamlines will be able to easily select between the standard x-rays originated by the standard electron bunches and the short $\mathrm{x}$-ray pulses originated by the special electron bunches carrying a time-dependent transverse correlation. Furthermore, two different modes of operation can simultaneously co-exist without degrading the x-ray flux and brightness and the x-ray pulse duration delivered in each mode. It has been shown that obtaining the x-ray pulses with an r.m.s. duration less than one picosecond is feasible for hard- and soft x-ray synchrotrons using realistic machine parameters and realistic parameters for the rf deflecting cavities.

Stimulating discussions with T. Berenc, M. Borland, P. Kuske and V. Sajaev are gratefully acknowledged. M. Borland and V. Sajaev also helped in using elegant. This work was supported by the Office of Science of the U.S. Department of Energy under Contract No. DE-AC02$06 \mathrm{CH} 11357$.

[1] W. Ackermann, G. Asova, V. Ayvazyan, A. Azima, N. Baboi, J. Bahr, V. Balandin, B. Beutner, A. Brandt, A. Bolzmann, et al., Nature Photonics 1, 336 (2007), URL http://dx.doi.org/10.1038/nphoton.2007.76.

[2] P. Emma, R. Akre, J. Arthur, R. Bionta, C. Bostedt, J. Bozek, A. Brachmann, P. Bucksbaum, R. Coffee, F. J. Decker, et al., Nature Photonics 4, 641 (2010).

[3] T. Ishikawa, H. Aoyagi, T. Asaka, Y. Asano, N. Azumi, T. Bizen, H. Ego, K. Fukami, T. Fukui, Y. Furukawa, et al., Nature Photonics 6, 540 (2012), URL http://dx. doi.org/10.1038/nphoton.2012.141.

[4] E. Allaria, R. Appio, L. Badano, W. A. Barletta, S. Bassanese, S. G. Biedron, A. Borga, E. Busetto, D. Castronovo, P. Cinquegrana, et al., Nature Photonics 6, 699 (2012).

[5] (2015), URL http://www.helmholtz-berlin.de/user/ workshops/from-pico-to-femto.

[6] Y. D. Chuang, W. S. Lee, Y. F. Kung, A. P. Sorini, B. Moritz, R. G. Moore, L. Patthey, M. Trigo, D. H.Lu, P. S. Kirchmann, et al., Phys. Rev. Lett. 110, 127404 (2013).

[7] W. Lee, Y. Chuang, R. Moore, Y. Zhu, L. Patthey, M. Trigo, D. Lu, P. Kirchmann, O. Krupin, M. Yi, et al., Nature Comm. 3, 838 (2012).

[8] J. H. Lee, M. Wulff, S. Bratos, J. Petersen, L. Guerin, J. C. Leicknam, M. Cammarata, Q. Kong, J. Kim, K. B. Moller, et al., J. Am. Chem. Soc. 135, 3255 (2013).

[9] H. Wen, P. Chen, M. P. Cosgriff, D. A. Walko, J. H. Lee, C. Adamo, R. D. Schaller, J. F. Ihlefeld, E. M. Dufresne, D. G. Schlom, et al., Phys. Rev. Lett. 110, 037601 (2013).

[10] G. Vanko, A. Bordage, M. Papai, K. Haldrup, P. Glatzel, A. M. March, G. Doumy, A. Britz, A. Galler, T. Assefa, et al., J. Phys. Chem C 119, 5888 (2015).

[11] J. Feikes, K. Holldack, P. Kuske, and G. Wüstefeld, in Proc. of the 2004 European Particle Accelerator Conference, Lucerne, Switzerland (2004), p. 2290.

[12] J. Feikes, M. von Hartrott, G. Wüstefeld, A. Hoehl, R. Klein, R. Muller, and G. Ulm, in Proc. of the 2009 Particle Accelerator Conference, Vancouver, BC, Canada 
(2009).

[13] X. Huang, J. Safranek, J. Corbett, Y. Nosochkov, J. Sebek, and A. Terebilo, in Proc. of the 2007 Particle Accelerator Conference, Albuquerque, USA (2007), p. 1308.

[14] J. Feikes, P. Kuske, and G. Wüstefeld, in Proc. of the 2006 European Particle Accelerator Conference, Edinburgh, Scotland (2006), p. 157.

[15] G. Wüstefeld, A. Jankowiak, J. Knobloch, and M. Ries, in Proc. of the 2011 International Particle Accelerator Conference, San Sebastian, Spain (2011), p. 2936.

[16] R. W. Schoenlein, S. Chattopadhyay, H. H. W. Chong, T. E. Glover, P. A. Heimann, C. V. Shank, A. A. Zholents, and M. S. Zolotorev, Science 287, 2237 (2000).

[17] S. Khan, K. Holldack, T. Kachel, R. Mitzner, and T. Quast, Phys. Rev. Lett. 97, 074801 (2006).

[18] P. Beaud, S. L. Johnson, A. Streun, R. Abela, D. Abramsohn, D. Grolimund, F. Krasniqi, T. Schmidt, V. Schlott, and G. Ingold, Phys. Rev. Lett. 99, 174801 (2007).

[19] A. He, F. Willeke, and L. H. Yu, Phys. Rev. ST Accel. Beams 17, 040701 (2014).

[20] A. Zholents, P. Heimann, M. Zolotorev, and J. Byrd, Nucl. Instrum. Methods Phys. Res. A 425, 385 (1999).
[21] M. Borland, Phys. Rev. ST Accel. Beams 8, 074001 (2005).

[22] D. Attwood, Soft X-rays and Extreme Ultraviolet Radiation: Principles and Applications (Cambridge University Press, 2000).

[23] M. Borland and V. Sajaev, in Proc. of the 2005 Particle Accelerator Conference, Knoxwille, USA (2005), p. 3886.

[24] (2015), URL https://www1.aps.anl.gov/.

[25] (2015), URL https://www.helmholtz-berlin.de/ quellen/bessy/index_en.html.

[26] A. Lunin, I. Gonin, T. Khabiboulline, V. Yakovlev, and A. Zholents, in Proc. of the 27th Lnear Accelerator Conference, Geneva, Switzerland (2014), p. 966.

[27] Z. Conway, A. Barcikowski, S. Gerbick, M. Kedzie, M. Kelly, J. Kerby, S. Kim, S. Kutsaev, R. Murphy, A. Nassiri, et al., in Proc. of the 5th International Particle Accelerator Conference, Dresden, Germany (2014), p. 2595.

[28] M. Borland, Report LS-287, Advanced Photon Source, Argonne National Laboratory (2000). 